\title{
Radiological features of infantile glioblastoma and desmoplastic infantile tumors: British Columbia's Children's Hospital experience
}

\author{
Ahmad Bader, MD, ${ }^{1}$ Manraj Heran, MD, FRCPC, ${ }^{2}$ Christopher Dunham, MD, FRCPC, ${ }^{3}$ and \\ Paul Steinbok, MBBS, FRCSC ${ }^{1,4}$
}

Divisions of ${ }^{1}$ Neurosurgery and ${ }^{3}$ Anatomic Pathology, Department of Pathology and Laboratory Medicine, University of British Columbia; and 2Department of Radiology and 'Division of Pediatric Neurosurgery, British Columbia's Children's Hospital, University of British Columbia, Vancouver, British Columbia, Canada

\begin{abstract}
OBJECT Two of the more common infantile brain tumors, glioblastoma multiforme (GBM) and desmoplastic infantile tumors (DITs), can be difficult to distinguish on MRI. Both tumors occur in the supratentorial compartment and both have solid and cystic components. Differentiating between the 2 on MRI studies could assist the surgeon in discussions with family and child management. The authors report on their institutional experience with both tumors, focusing on radiographic features, especially the diffusion studies, which might be useful in distinguishing between infantile GBM and DIT.

METHODS A retrospective review was undertaken of all infantile brain tumors treated at British Columbia's Children's Hospital between 1982 and 2012, and cases of GBM and DIT were recorded. Only cases that had imaging were included in the study. A literature review was completed to identify reported cases of infantile GBM and DIT. Only reports that described or included radiological studies (particularly MRI) of the tumors were included. Certain radiographic features of the tumors were reviewed, including location, size, consistency, pattern of enhancement, and features on MR diffusion studies.
\end{abstract}

RESULTS Of 70 cases of infantile brain tumors, 2 GBM cases and 3 DIT cases (all 3 of which were desmoplastic infantile gangliogliomas [DIGs]) met the inclusion criteria. The radiological studies obtained in all 5 cases were reviewed by a neuroradiologist. All 5 patients had supratentorial tumors with cystic-solid consistency. Diffusion MRI studies showed restricted diffusion in the 2 GBM cases, but no evidence of restricted diffusion in the DIG tumors. The GBM tumors were heterogeneously enhancing, and the DIG tumors showed avid and homogeneous enhancement. The literature review revealed 29 cases of infantile GBM and 32 cases of DIG/DIT that met the inclusion criteria. The tumors were large in both groups. The tumors were cystic-solid in consistency in 10 of $30(33 \%)$ of GBM cases and 28 of $32(87.5 \%)$ of DIT cases. The contrast enhancement was heterogeneous in 9 of $30(30 \%)$ GBM cases, and it was homogeneous and avid in 27 of $32(84 \%)$ of DIT cases. Diffusion studies were recorded in 2 published infantile GBM cases, and in both of them diffusion was restricted. The authors only found 1 report that discussed DIG tumor features on MR diffusion studies, but the interpretation was difficult and unclear.

CONCLUSIONS Magnetic resonance imaging, especially diffusion-weighted imaging, may be a useful aid in distinguishing between infantile GBM and DIT tumors, with infantile GBM demonstrating restricted diffusion.

http://thejns.org/doi/abs/10.3171/2014.10.PEDS13634

KEY WORDS infantile glioblastoma; desmoplastic infantile ganglioglioma; desmoplastic infantile astrocytoma; congenital brain tumors; brain magnetic resonance imaging; diffusion; oncology 
B RAIN tumors are the second most frequent type of cancer occurring in children. The peak age of incidence in children is between 5 and 8 years. ${ }^{25,28}$ Between $1.3 \%$ and $11 \%$ of pediatric brain tumors occur in the 1st year of life, with the majority of tumors occurring supratentorially. ${ }^{5,17,25,28,33}$ The pathological types of infantile brain tumors include malignant tumors such as primitive neuroectodermal tumors (PNETs), atypical teratoid and rhabdoid tumors, and glioblastoma multiforme (GBM). However, benign tumors such as desmoplastic infantile tumors (DITs)-i.e., desmoplastic infantile ganglioglioma (DIG) and desmoplastic infantile astrocytoma-do occur in infancy. Prognosis depends on the tumor pathology, with malignant tumors almost universally doing poorly with respect to both mortality rates and long-term outcome of survivors. ${ }^{13}$ If it was possible to predict with high certainty the pathological features of an infantile brain tumor based on radiographic findings and, in particular, whether the tumor was malignant, this could assist in discussions with the family and the management of the child.

On standard T1- and T2-weighted MRI scans performed with and without contrast, 2 of the more common supratentorial infantile tumors (namely GBM, which is highly malignant, and DIG/DIT, which is benign) may be difficult to distinguish. Both tumor types may demonstrate solid and cystic components, with irregular enhancement, peritumoral edema, and apparent invasion of adjacent brain. Although diffusion-weighted imaging (DWI) is often performed in the assessment of brain tumors, the diffusion characteristics of these infantile tumors have been reported rarely in the literature.

We hypothesized that DWI would show restricted diffusion in the solid components of infantile GBM, but either no restriction or increased diffusion in the infantile DIG. The purpose of this study was to review the MRI features of both tumors, with attention to the diffusion imaging to see if the findings on diffusion MRI scans could distinguish between the 2 tumor types.

\section{Methods}

A retrospective review of the prospectively maintained neurosurgery and brain tumor databases at British Columbia's Children's Hospital (BCCH) and the British Columbia Cancer Agency was done to identify infantile brain tumors diagnosed between 1982 and 2012. Infants were defined as patients younger than 1 year at the time of diag- nosis. From the total list of infantile brain tumors, cases of infantile GBM, DIG, and DIT were extracted.

For patients to be included in this study, they had to have a pathologically confirmed GBM (WHO Grade IV), DIG, or DIT (both WHO Grade I) and have undergone MRI (including DWI) as part of their evaluation. The patient's clinical presentation, neuroradiological studies, and individual treatments, including surgical intervention, chemotherapy, and radiation therapy, were recorded. The radiology reports were reviewed. In addition, the radiological studies were re-reviewed for this study by 2 neuroradiologists at $\mathrm{BCCH}$. Factors on imaging that were reviewed included the following: tumor location, tumor size, presence and size of cyst, contrast-enhancement pattern, and presence or absence of restricted diffusion on MRI diffusion studies, with restricted diffusion defined as a reduction in apparent diffusion coefficient (ADC) values in the lesion by greater than $10 \%$ when compared with normal contralateral parenchyma.

The diagnosis of GBM, DIG, and DIT was based on the WHO 2007 classification of CNS tumors. All slides were reviewed, which included immunohistochemistry, to exclude alternative diagnoses.

A literature review of all published case reports and series of infantile and congenital GBM and DIG/DITs was undertaken through a search. Key words used included glioblastoma, infants, GBM, desmoplastic infantile tumors, DIG, desmoplastic infantile ganglioglioma, and MRI. Case series that included imaging findings in their reports were included. Imaging studies included head ultrasound and CT and/or MRI scan of the brain. In those reports we reviewed the location of the tumor; its consistency, size, contrast enhancement pattern; and the diffusion characteristics on DWI if these were included.

\section{Results}

There were a total of 70 cases of infantile brain tumors treated at BCCH between 1982 and 2012. Of these, 4 cases were coded as GBM and 3 as DIG. Of the 4 patients with GBM, 2 cases were excluded from this study. One was excluded because the infant died shortly after admission to hospital before any radiological studies were performed, with the diagnosis made on postmortem autopsy. The other case was excluded because the tumor's pathological findings "matured" to pleomorphic xanthoastrocytoma on repeat surgery for tumor progression. This case has been reported previously. ${ }^{45}$

TABLE 1. Characteristics of 2 GBM and 3 DIG cases on MRI*

\begin{tabular}{cccccc}
\hline \multirow{2}{*}{$\begin{array}{c}\text { Case } \\
\text { No. }\end{array}$} & Supratentorial Location & Cystic-Solid & ADC Restriction & Enhancement & Pathological \\
\cline { 2 - 5 } & Findings \\
\hline 1 & Yes & Yes & Yes & Heterogeneous (solid) & GBM \\
\hline 2 & Yes & Yes & Yes & Heterogeneous (solid) & GBM \\
\hline 3 & Yes & Yes & No & Avid (solid) & DIT \\
\hline 4 & Yes & Yes & No & Avid (solid) & DIT \\
\hline 5 & Yes & Yes & No & Avid (solid) & DIT \\
\hline
\end{tabular}

* The BCCH experience. Cases 1 and 2 were infantile GBM; Cases 3-5 were DITs. 

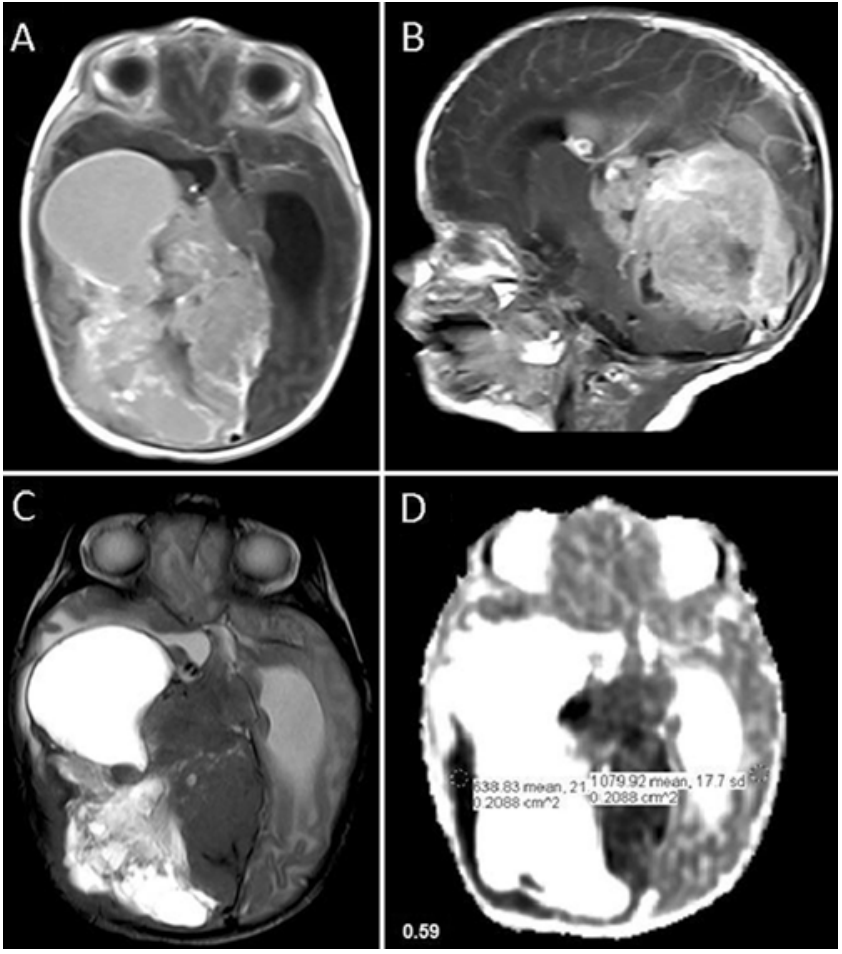

FIG. 1. Case 1. Admission MR images. A: Axial T1-weighted image obtained post-Gd shows the large cystic and solid tumor with associated heterogeneous enhancement of the solid part. B: Sagittal T1-weighted image obtained post-Gd showing the extent and size of the cystic component of the tumor. The tumor involves virtually the whole right hemisphere, sparing the frontal region. C: Axial T2-weighted image showing the large cystic component of the tumor. The cystic fluid has higher signal than CSF. D: An ADC map showing the restricted diffusion of the solid component of the tumor.

The neuroradiological studies of the 2 pathologically proven cases of GBM and the 3 cases of DIG were reviewed. Both cases of GBM were supratentorial, large, involved multiple lobes, had prominent large cystic components, had heterogeneous contrast enhancement, and had a wide area that was adjacent to the overlying dura mater. Both cases demonstrated restricted diffusion of the solid tumoral component on DWI.

In 2 of the 3 DIG cases, the tumors were large, located in the supratentorial compartment, had a large cystic component, and a peripheral solid component that avidly enhanced on contrast studies. The third tumor was predominantly solid with a small cyst. The solid tumor nodule demonstrated avid enhancement on postcontrast imaging in all 3 cases. Also in all 3 cases, the tumor nodule was peripherally located and was adjacent to the overlying dura. There was normal diffusion (i.e., no evidence of restricted diffusion) of the solid part on DWI (Table 1).

The literature review revealed 18 relevant publications on infantile GBM, of which 12 were case reports and 6 were case series. ${ }^{5,6,10,12,17,18,21,24-26,28,31,33-36,41,42}$ From these publications, there were 29 cases of GBM that met our inclusion criteria. The patients underwent either CT scans or MRI based on the patient's age and the clinical presentation. The tumor was located in the supratentorial

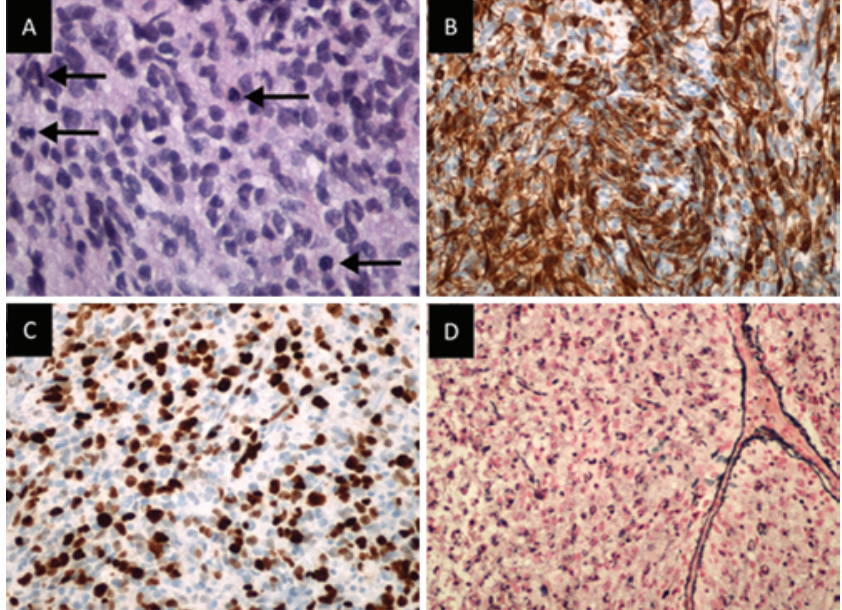

FIG. 2. Case 1. Photomicrographs showing histopathological features of specimens obtained at surgery. A: Routine $\mathrm{H}$ \& $\mathrm{E}$ staining reveals enlarged, pleomorphic, and hyperchromatic tumor nuclei associated with relatively little fibrillary cytoplasm. Mitoses (arrows) are numerous. B: The GFAP staining is strong. C: The Ki 67 proliferative index is high. D: The reticulin staining is limited to the blood vessel at right. Original magnification $\times 400(A), \times 200(B-D)$. Figure is available in color online only.

space in all cases. It was large in all cases, cystic-solid in 10 cases, ${ }^{6,10,12,17,18,24,28,31,32}$ had heterogeneous enhancement in 9 cases. . $^{5,12,17,18,21,31,35}$ Contrast was not mentioned or not given in 19 cases. ${ }^{5,6,8,10,12,16,18,24-26,28,34,36,42}$ Marked enhancement with heterogeneous intratumoral signal due to hemorrhage was reported in 1 case..$^{33}$ Two publications included the results of DWI, and both tumors had restricted diffusion in the solid component..$^{10,34}$

Nineteen reports of desmoplastic tumors were identified and reviewed. From those, 9 publications were single case reports and 10 were case series with a minimum of 2 cases and a maximum of 8 cases. A total of 38 cases were collected. Six cases from 1 publication were excluded because the imaging findings, which were included, did not fit the description by the authors of the radiological find-
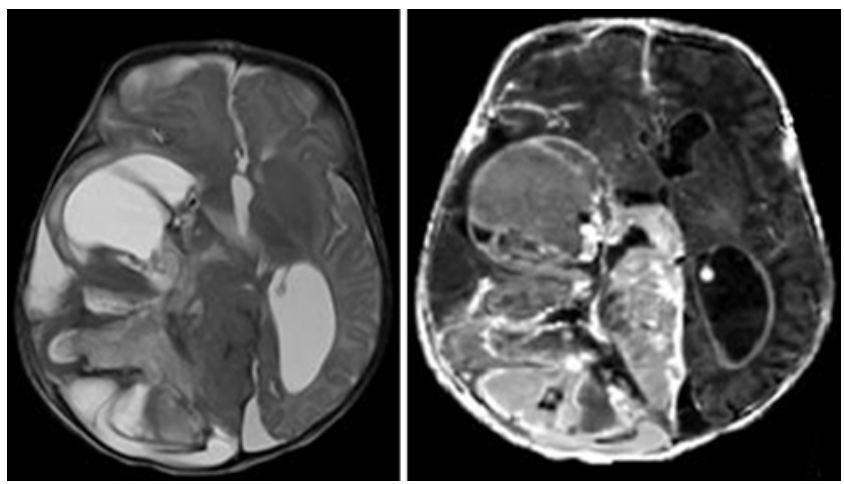

FIG. 3. Case 1. Postoperative MRI studies obtained 1 week after diagnosis, biopsy, and decompression of the cyst. Left: Axial T2-weighted image showing the progression of the solid component, and recurrence of the cyst. Right: Axial T1-weighted post-Gd image showing more avid heterogeneous enhancement of the tumor, as well as subependymal enhancement of the lateral ventricles. 

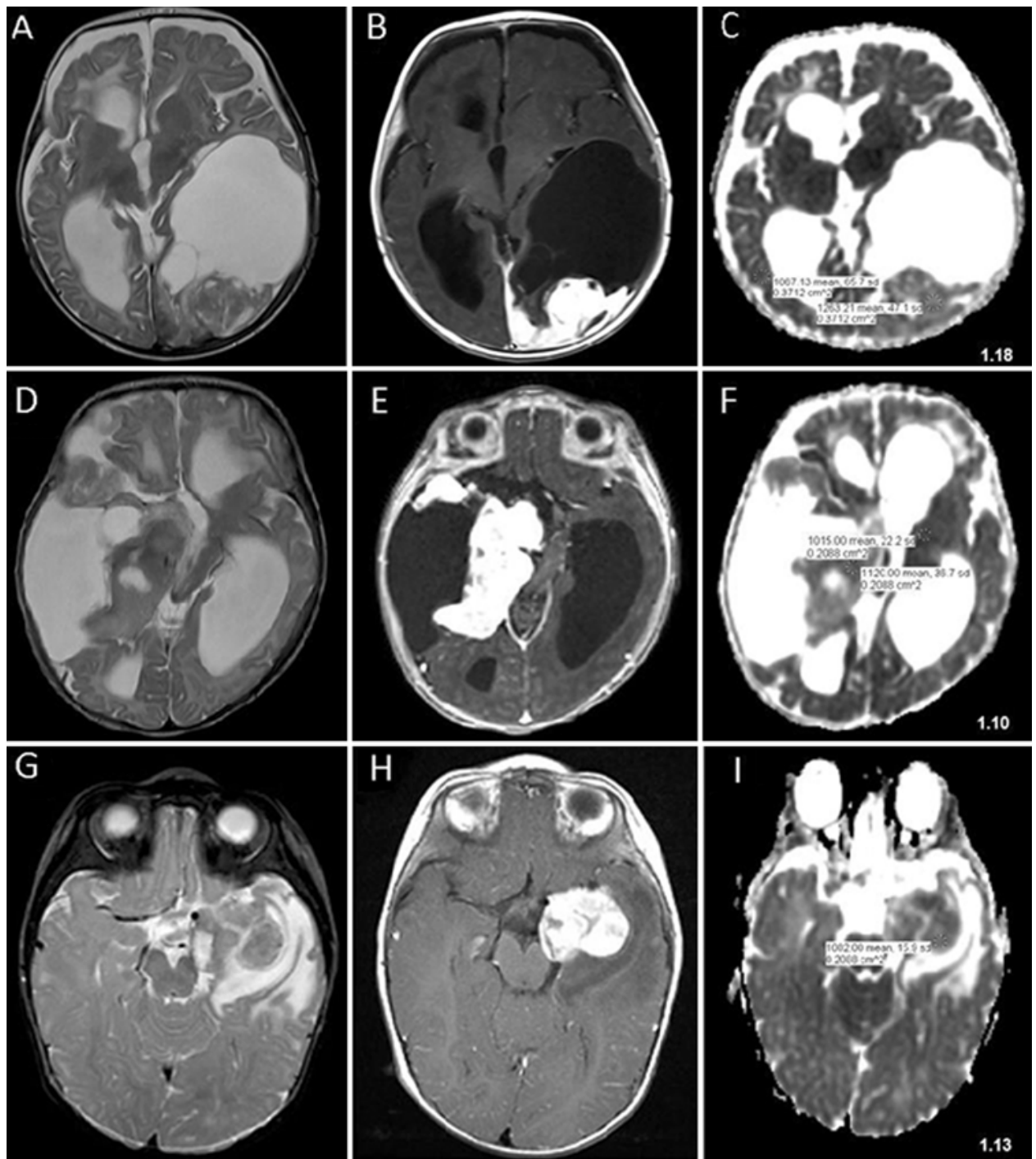

FIG. 4. Admission MRI features in 3 patients (Cases 3-5) with DITs. A-C: Case 3. Axial T2-weighted, T1-weighted post-Gd, and $A D C$ map images, respectively, showing cystic-solid tumor, avidly enhancing solid component, and normal diffusibility. D-F: Case 4. Axial T2-weighted, T1-weighted post-Gd, and ADC map images, respectively, showing cystic-solid tumor, avidly enhancing solid component, and normal diffusibility. G-I: Case 5. Axial T2-weighted, T1-weighted post-Gd, and ADC map images, respectively, showing a predominantly left temporal solid tumor, avidly enhancing, and with normal diffusibility.

ings. ${ }^{23}$ The tumor was found to be large and located in the supratentorial compartment in all cases. Cystic-solid consistency of the tumor was either reported by authors or dis-

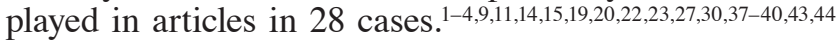
A homogeneously and avidly enhancing solid nodule was the most common finding among those cases (27 of 32). ${ }^{1,3,4,9,11,14,15,19,22,27,30,38-40,43}$ Either no contrast was given or the pattern of enhancement was interpreted as heterogeneous in 7 cases. ${ }^{2,15,20,23,37,44}$ Only 1 publication reported the features of DIG on DWI; ${ }^{37}$ however, the description was vague. The tumor was described as having "hyperintense signal on diffusion weighted images," but did not describe the imaging pattern on an ADC map because the ADC characteristics were not available for review. ${ }^{37}$

Examples of a GBM and a DIG from our series are presented below.

\section{Illustrative Cases \\ Case 1: GBM}

A 3-week-old girl born after an uneventful pregnancy and normal vaginal delivery presented with macrocephaly, bulging fontanelle, and increasing irritability. There had been a dramatic increase in the head circumference between birth and 3 weeks. A head ultrasound showed a large heterogeneous lesion centered in the right hemisphere. This lesion consisted of large cyst and a fairly large solid component. Subsequently, a brain MRI study was obtained. This showed a large cystic and solid tumor measuring $10 \times 11 \times 8 \mathrm{~cm}$ that was centered in the right cerebral hemisphere. The cystic component showed homogeneous hyperintense signal on T1- and T2-weighted images. The solid component was hypointense to brain on 
$\mathrm{T} 1$ and $\mathrm{T} 2$ and demonstrated restricted diffusion on the ADC map. The solid component had heterogeneous enhancement (Fig. 1).

A right parietooccipital craniotomy for cyst drainage and tissue diagnosis was done. No attempt was made to resect the tumor because the lesion was very vascular, friable, and necrotic.

Pathological assessment revealed GBM (WHO Grade IV). The tumor cells were disposed in patternless sheets. There was evidence of pseudopalisading necrosis and microvascular proliferation. The Ki 67 proliferative index was high. The tumor cells were negative for IDH1 on immunohistochemical investigation (Fig. 2).

The patient did well postoperatively, and was discharged home. However, she presented 1 week later with a bulging fontanelle, poor feeding, and increasing irritability. Repeat brain MRI showed recurrence of the cyst, blood clot in the cyst, further enlargement of the solid component, and evidence of leptomeningeal dissemination along the lining of the ventricles and along the spinal cord (Fig. 3). Palliative measures were recommended and the child died 1 week later.

\section{Case 3: DIG}

A 3-month-old girl presented with increasing head circumference. Her examination confirmed full anterior fontanelle; otherwise she was neurologically intact. Brain MRI showed a large hemispheric intraaxial solid-cystic mass with the solid part centered in the left occipital region. An avidly enhancing tumor nodule was seen. No restricted diffusion was seen on DWI (Fig. 4A-C).

The patient underwent an uneventful left occipital craniotomy and gross-total resection of the tumor. The cystic component was decompressed. The dura overlying tumor was grossly involved.

A DIG was confirmed pathologically. The tumor was composed of short fascicles of spindle cells with oval nuclei and poorly defined cell borders in a collagenous background. The tumor stained positive for glial fibrillary acidic protein (GFAP), synaptophysin, neuron-specific nuclear protein, neurofilament, neuron-specific enolase, and S100. The Ki 67 proliferative index was 10\%-11\% (Fig. 5).

The patient did well postoperatively, and was discharged home with no complications. On follow-up examination she was found to have a right visual field defect (homonymous hemianopia), but otherwise she was developing well, with no other deficits. Her postoperative MRI studies showed a slight amount of enhancement close to the junction of the sigmoid and left transverse sinus. This was thought to be a small residual lesion. Her MRI studies have shown no recurrence of her disease at 3 years after resection.

\section{Discussion}

In this report we outline the MRI features of 2 types of infantile brain tumors, infantile GBM and DIT. These tumors are rare during infancy. Distinguishing between them can be difficult, but it is still important because one tumor is histologically benign and the other is malignant; differentiating these tumors prior to surgery may help

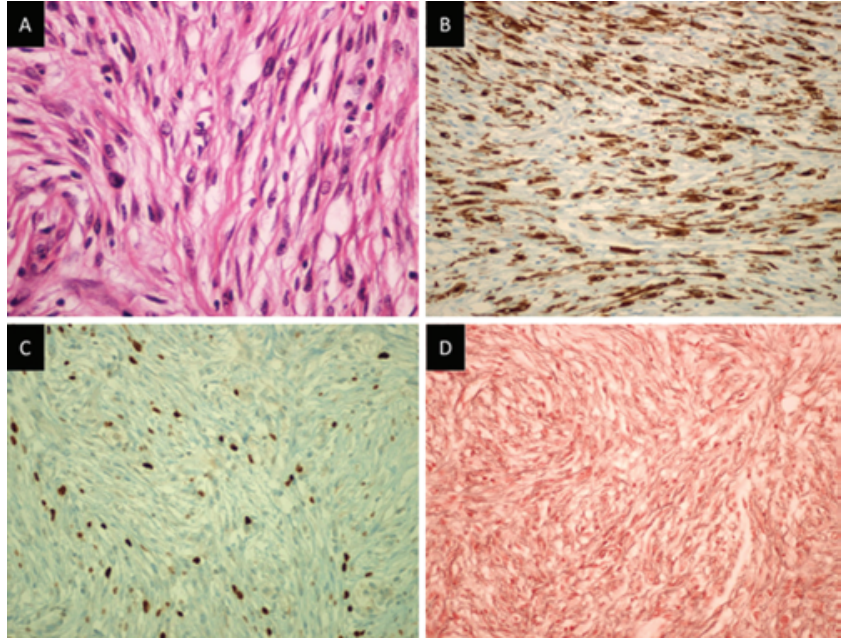

FIG. 5. Case 3. Photomicrographs showing histopathological features of specimens obtained at surgery. A: Routine H \& E staining reveals spindled tumor cells with thin, wispy, cytoplasmic fibers among thin connective tissue (i.e., reticulin) fibers. B: The GFAP staining highlights several spindled tumor cells. C: The Ki 67 proliferative index is moderate in this spindled tumor cell area. D: The reticulin staining reveals several thin fibers. Original magnification $\times 400(A), \times 200(B-D)$. Figure is available in color online only.

guide treatment, predict surgery-related morbidity, and assist in family counseling. Infantile GBM tumors are usually highly vascular lesions with a potential risk of significant blood loss intraoperatively. Predicting pathological findings preoperatively may help the surgeon plan for only tumor biopsy, chemotherapy, and then second look/resection surgery instead of attempting complete resection, which can be highly morbidity-producing to patients.

Our series showed that both types of tumors appeared large on MRI studies. In the majority of patients, they occupied 1-2 lobes or even the whole hemisphere, were located in the supratentorial space, had a cystic-solid consistency, and had a wide area that was adjacent to the dura. However, GBM tumors had heterogeneous enhancement, and the solid part showed restricted diffusion on MR diffusion studies. On the other hand, the DIG tumors had avid enhancement and did not demonstrate restricted diffusion on the DWI.

Our literature review revealed similar results. However, MR diffusion studies were rarely reported or used to evaluate these lesions. Two reports included diffusion studies of their patients with infantile GBM, and in both of them diffusion was restricted..$^{10,34}$ One report provided a description of the appearance of a DIG tumor on DWI, but interpretation was limited. ${ }^{37}$

Preoperative DWI provides valuable information about the tumor cellularity and structural integrity by measuring differences in the ADC. ${ }^{29}$ It is widely used in the assessment of brain tumors, ischemic events, and CNS infections in both adult and pediatric patients. It is easy to perform and requires very little scanning time.

In this report we focused on the diffusion characteristics of 2 common infantile tumors. Other malignant infan- 
tile tumors such as PNET are known to have restricted diffusion on DWI, and previous reports have discussed this feature. In 1 report of 12 supratentorial and infratentorial PNETs, all cases showed restricted diffusion on DWI?

\section{Conclusions}

We present 2 cases of pathologically proven infantile GBM and 3 cases of DIG. Each group had very suggestive MRI features. The pattern of enhancement and appearance on diffusion studies was very predictive for each group. The information provided by the diffusion studies may be of clinical significance to neurosurgeons. It adds information that may be useful for surgical planning, family counseling preoperatively, and predicting potential intraoperative morbidities.

\section{Acknowledgment}

We thank Dr. Michael Sargent, Department of Radiology, $\mathrm{BCCH}$, for re-reviewing the patients' radiological studies.

\section{References}

1. Al-Kharazi K, Gillis C, Steinbok P, Dunham C: Malignant desmoplastic infantile astrocytoma? A case report and review of the literature. Clin Neuropathol 32:100-106, 2013

2. Alexiou GA, Stefanaki K, Sfakianos G, Prodromou N: Desmoplastic infantile ganglioglioma: a report of 2 cases and a review of the literature. Pediatr Neurosurg 44:422-425, 2008

3. Balaji R, Ramachandran K: Imaging of desmoplastic infantile ganglioglioma: a spectroscopic viewpoint. Childs Nerv Syst 25:497-501, 2009

4. Beppu T, Sato Y, Uesugi N, Kuzu Y, Ogasawara K, Ogawa A: Desmoplastic infantile astrocytoma and characteristics of the accompanying cyst. Case report. J Neurosurg Pediatr 1:148-151, 2008

5. Brat DJ, Shehata BM, Castellano-Sanchez AA, Hawkins C, Yost RB, Greco C, et al: Congenital glioblastoma: a clinicopathologic and genetic analysis. Brain Pathol 17:276-281, 2007

6. Buetow PC, Smirniotopoulos JG, Done S: Congenital brain tumors: a review of 45 cases. AJR Am J Roentgenol 155:587-593, 1990

7. Chawla A, Emmanuel JV, Seow WT, Lou J, Teo HE, Lim CC: Paediatric PNET: pre-surgical MRI features. Clin Radiol 62:43-52, 2007

8. Chuang YM, Guo WY, Ho DM, Wong TT, Hung JH, Chen SJ, et al: Skew ocular deviation: a catastrophic sign on MRI of fetal glioblastoma. Childs Nerv Syst 19:371-375, 2003

9. Darwish B, Arbuckle S, Kellie S, Besser M, Chaseling R: Desmoplastic infantile ganglioglioma/astrocytoma with cerebrospinal metastasis. J Clin Neurosci 14:498-501, 2007

10. Donmez FY, Aslan H, Coban G, Ozen O, Agildere M: Multimodal MR imaging findings of a congenital glioblastoma multiforme. Childs Nerv Syst 28:1831-1833, 2012

11. Duffner PK, Burger PC, Cohen ME, Sanford RA, Krischer JP, Elterman R, et al: Desmoplastic infantile gangliogliomas: an approach to therapy. Neurosurgery 34:583-589, 1994

12. Duffner PK, Krischer JP, Burger PC, Cohen ME, Backstrom JW, Horowitz ME, et al: Treatment of infants with malignant gliomas: the Pediatric Oncology Group experience. J Neurooncol 28:245-256, 1996

13. Dunham C, Pillai S, Steinbok P: Infant brain tumors: a neuropathologic population-based institutional reappraisal. Hum Pathol 43:1668-1676, 2012
14. Finizio FS: CT and MRI aspects of supratentorial hemispheric tumors of childhood and adolescence. Childs Nerv Syst 11:559-567, 1995

15. Gu S, Bao N, Yin MZ: Combined fontanelle puncture and surgical operation in treatment of desmoplastic infantile astrocytoma: case report and a review of the literature. J Child Neurol 25:216-221, 2010

16. Heckel S, Favre R, Gasser B, Christmann D: Prenatal diagnosis of a congenital astrocytoma: a case report and literature review. Ultrasound Obstet Gynecol 5:63-66, 1995

17. Hou LC, Bababeygy SR, Sarkissian V, Fisher PG, Vogel H, Barnes P, et al: Congenital glioblastoma multiforme: case report and review of the literature. Pediatr Neurosurg 44:304-312, 2008

18. Jung WH, Choi S, Oh KK, Chi JG: Congenital glioblastoma multiforme-report of an autopsy case. J Korean Med Sci 5:225-231, 1990

19. Kesavadas C, Sonwalker H, Thomas B, Gupta AK, Radhakrishnan VV: Atypical MRI appearance of desmoplastic infantile ganglioglioma. Pediatr Radiol 35:1024-1026, 2005

20. Komori T, Scheithauer BW, Parisi JE, Watterson J, Priest JR: Mixed conventional and desmoplastic infantile ganglioglioma: an autopsied case with 6-year follow-up. Mod Pathol 14:720-726, 2001

21. Kotecha RS, Burley K, Junckerstorff RC, Lee S, Phillips MB, Cole $\mathrm{CH}$, et al: Chemotherapy increases amenability of surgical resection in congenital glioblastoma. Pediatr Hematol Oncol 29:538-544, 2012

22. Louis DN, von Deimling A, Dickersin GR, Dooling EC, Seizinger BR: Desmoplastic cerebral astrocytomas of infancy: a histopathologic, immunohistochemical, ultrastructural, and molecular genetic study. Hum Pathol 23:1402-1409, 1992

23. Mallucci C, Lellouch-Tubiana A, Salazar C, Cinalli G, Renier D, Sainte-Rose C, et al: The management of desmoplastic neuroepithelial tumours in childhood. Childs Nerv Syst 16:8-14, 2000

24. Mazzone D, Magro G, Lucenti A, Grasso S: Report of a case of congenital glioblastoma multiforme: an immunohistochemical study. Childs Nerv Syst 11:311-313, 1995

25. Milano GM, Cerri C, Ferruzzi V, Capolsini I, Mastrodicasa E, Genitori L, et al: Congenital glioblastoma. Pediatr Blood Cancer 53:124-126, 2009

26. Nakayama K, Nakamura Y: Localization of congenital glioblastomas in the Japanese: a case report and review of the literature. Childs Nerv Syst 18:149-152, 2002

27. Nikas I, Anagnostara A, Theophanopoulou M, Stefanaki K, Michail A, Hadjigeorgi C: Desmoplastic infantile ganglioglioma: MRI and histological findings case report. Neuroradiology 46:1039-1043, 2004

28. Nomura Y, Yasumoto S, Yanai F, Akiyoshi H, Inoue T, Nibu $\mathrm{K}$, et al: Survival and late effects on development of patients with infantile brain tumor. Pediatr Int 51:337-341, 2009

29. Park SM, Kim HS, Jahng GH, Ryu CW, Kim SY: Combination of high-resolution susceptibility-weighted imaging and the apparent diffusion coefficient: added value to brain tumour imaging and clinical feasibility of non-contrast MRI at 3 T. Br J Radiol 83:466-475, 2010

30. Phi JH, Koh EJ, Kim SK, Park SH, Cho BK, Wang KC: Desmoplastic infantile astrocytoma: recurrence with malignant transformation into glioblastoma: a case report. Childs Nerv Syst 27:2177-2181, 2011

31. Rivero-Garvía M, Márquez-Rivas J, Rueda-Torres AB, Pascual-Argente D, Ramírez G: Treatment of glioblastoma multiforme with high doses of carmustine intracavitary, in an infant. Childs Nerv Syst 28:747-750, 2012

32. Sakamoto K, Kobayashi N, Ohtsubo H, Tanaka Y: Intracranial tumors in the first year of life. Childs Nerv Syst 2:126129, 1986 
33. Seker A, Ozek MM: Congenital glioblastoma multiforme. Case report and review of the literature. J Neurosurg 105 (6 Suppl):473-479, 2006

34. Severino M, Schwartz ES, Thurnher MM, Rydland J, Nikas I, Rossi A: Congenital tumors of the central nervous system. Neuroradiology 52:531-548, 2010

35. Shimamura N, Asano K, Ogane K, Yagihashi A, Ohkuma $\mathrm{H}$, Suzuki S: A case of definitely congenital glioblastoma manifested by intratumoral hemorrhage. Childs Nerv Syst 19:778-781, 2003

36. Singhal RM, Jain M, Tanwar RK, Raman NV: Congenital glioblastoma. Indian J Pediatr 61:733-736, 1994

37. Sperner J, Gottschalk J, Neumann K, Schörner W, Lanksch WR, Scheffner D: Clinical, radiological and histological findings in desmoplastic infantile ganglioglioma. Childs Nerv Syst 10:458-463, 1994

38. Sugiyama K, Arita K, Shima T, Nakaoka M, Matsuoka T, Taniguchi E, et al: Good clinical course in infants with desmoplastic cerebral neuroepithelial tumor treated by surgery alone. J Neurooncol 59:63-69, 2002

39. Tamburrini G, Colosimo C Jr, Giangaspero F, Riccardi R, Di Rocco C: Desmoplastic infantile ganglioglioma. Childs Nerv Syst 19:292-297, 2003

40. Tenreiro-Picon OR, Kamath SV, Knorr JR, Ragland RL, Smith TW, Lau KY: Desmoplastic infantile ganglioglioma: CT and MRI features. Pediatr Radiol 25:540-543, 1995

41. Tewari MK, Sharma BS, Mahajan RK, Khosla VK, Mathuriya SN, Pathak A, et al: Supratentorial tumours in infants.

Childs Nerv Syst 10:172-175, 1994
42. Thankamony A, Harlow FH, Ponnampalam J, Clarke P: Congenital brain tumour mimicking fetal intracranial haemorrhage. J Obstet Gynaecol 27:314-317, 2007

43. Trehan G, Bruge H, Vinchon M, Khalil C, Ruchoux MM, Dhellemmes P, et al: MR imaging in the diagnosis of desmoplastic infantile tumor: retrospective study of six cases. AJNR Am J Neuroradiol 25:1028-1033, 2004

44. VandenBerg SR: Desmoplastic infantile ganglioglioma and desmoplastic cerebral astrocytoma of infancy. Brain Pathol 3:275-281, 1993

45. Yang MM, Singhal A, Rassekh SR, Yip S, Eydoux P, Dunham C: Possible differentiation of cerebral glioblastoma into pleomorphic xanthoastrocytoma: an unusual case in an infant. J Neurosurg Pediatr 9:517-523, 2012

\section{Author Contributions}

Conception and design: all authors. Acquisition of data: all authors. Analysis and interpretation of data: all authors. Drafting the article: all authors. Critically revising the article: all authors. Reviewed submitted version of manuscript: all authors. Approved the final version of the manuscript on behalf of all authors: Steinbok. Administrative/technical/material support: Bader. Study supervision: Steinbok, Bader.

\section{Correspondence}

Paul Steinbok, Division of Pediatric Neurosurgery, BC Children's Hospital, 4480 Oak St., Rm. K3-159, Vancouver, BC V6H 3V4, Canada.email: psteinbok@cw.bc.ca. 